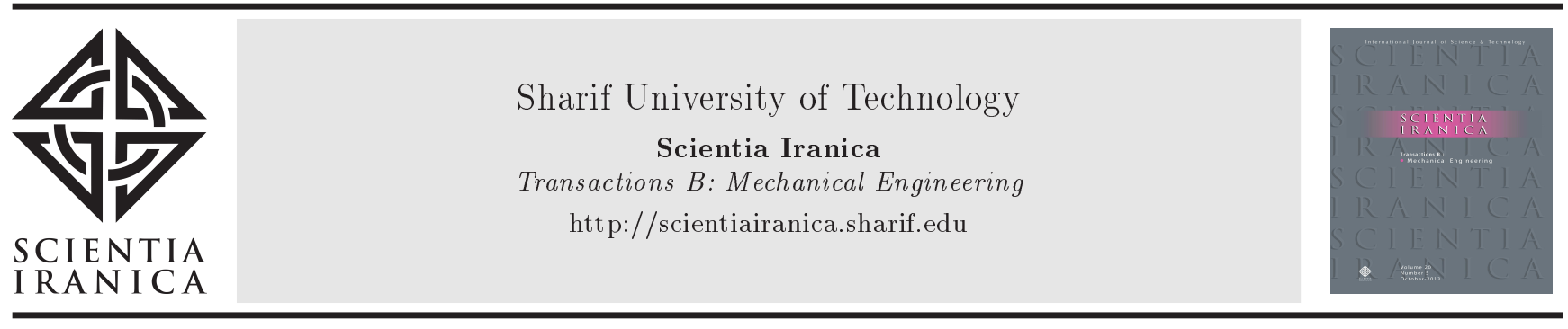

\title{
An approximate methodology to simulate combined conduction-radiation heat transfer for multi-layer insulator
}

\author{
A. Torabi ${ }^{a, b}$ A. Abedian ${ }^{b, *}$, and M.A. Farsi ${ }^{a}$ \\ a. Aerospace Research Institute (Ministry of Science, Research and Technology),14665-834, Tehran, Iran. \\ b. Department of Aerospace Engineering, Sharif University of Technology, Tehran, P.O. Box 11365-11155, Iran.
}

Received 9 March 2019; received in revised form 19 May 2019; accepted 12 October 2019

\author{
KEYWORDS \\ Combined conduction- \\ radiation; \\ Reflective multi-layer \\ insulator; \\ Approximate \\ methodology; \\ Effective thermal \\ conductivity; \\ Absorption area.
}

\begin{abstract}
A quasi-analytical methodology was developed to model combined conductionradiation heat transfer through the thickness of a reflective multi-layer insulator. This methodology was validated based on the experimental result. It can be applied to the initial design of high-temperature multi-layer insulators. Traditionally, radiation thermal conductivity approximation was employed for the initial stages of design. Despite the acceptable accuracy of this approach in steady state cases, it yields some unacceptable errors when thermal load is transient. It was shown that the older version of this methodology could not predict maximum temperature and time of occurrences by acceptable margins. The developed model originated from the radiation thermal conductivity approximation. Unlike the primitive one, the developed model shows acceptable performance in transient cases. This model was developed with emphasis on thermal emittance through the thickness of the insulator. It can predict the maximum temperature of a structure and its occurrence time with an error less than $4 \%$.
\end{abstract}

(C) 2020 Sharif University of Technology. All rights reserved.

\section{Introduction}

Different approaches are used to simulate radiation heat transfer through the thickness of hightemperature insulators [1]. Petrov compared three different approaches in this field: two-flux, diffusion, and effective thermal conductivity [2]. Effective thermal conductivity approximation is inherently simple and does not require radiation heat transfer equation. This approach is popular, especially for researches in the field of design or sizing of the high-temperature insulators [3,4]. For example, an ordinary equation is proposed for the radiative thermal conductivity of

*. Corresponding author.

E-mail address: abedian@sharif.ir (A. Abedian) space shuttle tiles [5]. Streed also developed and utilized an equation to calculate radiative thermal conductivity of the multi-layer insulator. This model was verified experimental based on results in the steady state case [6]. Radiation heat transfer is dominant, especially through the thickness of porous insulators [7]. Lacroix evaluated the effective thermal conductivity of porous matrices experimentally and analytically [8].

However, effective thermal conductivity does not provide accurate results in transient cases. It appears that this inaccuracy originates from the fact that the effective thermal conductivity approach does not consider local optical properties of tiny elements through the thickness of the insulator. Diffusion and two-flux approaches are more accurate in the transient case [2]. Diffusion approach simulates radiative heat transfer in optically thick insulators well $[9,10]$. This approach is normally used to calculate the effective thermal 
conductivity of different insulators [11]. However, twoflux approach can be applied to both cases of optically thin or thick insulators. Two-flux approach is widely used to simulate the heat transfer behavior of fibrous insulation $[12,13]$.

The reflective multilayer materials could be used as an insulator within integrated Thermal Protection Systems (TPS) [14]. Different theoretical models were proposed to simulate radiation heat transfer in reflective multilayer insulators [15-17]. Furthermore, Daryabeigi used both two-flux and diffusion approximations to simulate radiation heat transfer through a multilayer insulator utilized in an integrated TPS [17$19]$.

The two-flux approach yields a boundary value problem, which normally should be solved numerically $[20,21]$. If the insulator does not absorb radiation energy, the boundary value problem will be simplified and can be solved analytically. An analytical solution yields an equation for radiative thermal conductivity [22]. This methodology was utilized to propose the effective thermal conductivity of space shuttle tiles for steady-state cases [23].

In this paper, a quasi-analytical model is developed to simulate heat transfer through a hightemperature reflective multilayer insulator. Due to the inherent simplicity of this model, it is suitable for the design process of high-temperature insulators. First, for simplification, it is assumed that the absorption mechanism does not have a considerable effect on the thermal response of the insulator. This assumption yields a simplified two-flux boundary value problem, which can be solved analytically. This model is applied to a transient case and results are compared with the experimental results. It is shown that the thermal response of this model is not accurate in this case. In the following, the absorption mechanism is given in a simplified form and applied to the model, yielding a quasi-analytical methodology that reduces the susceptibility of the model to error for the transient case.

\section{Mathematical model}

Heat transfer through a multi-layer insulator could be modeled by the heat transfer equation. Conduction and radiation heat transfer modes of heat transfer could be separated, as illustrated in Eq. (1):

$$
\rho C_{P} \frac{\partial T}{\partial t}=K \frac{\partial^{2} T}{\partial x^{2}}-\frac{d q_{r}}{d x}
$$

It is considered that energy is transmitted to the external hot side of the insulator $(x=0)$ by convection. However, on the cold side of the insulator $(x=$ $L$ ), energy is transmitted to the substrate from the interface of structure and insulator, as presented in Eq. (2):

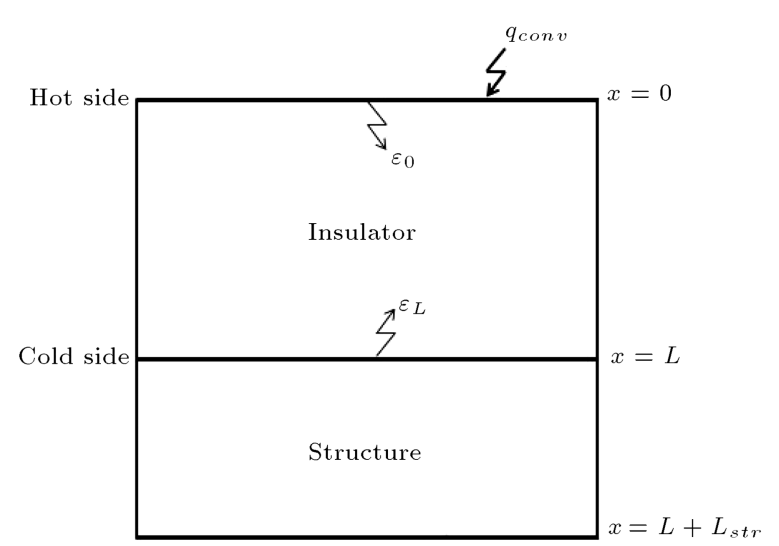

Figure 1. Boundary conditions of High Temperature Insulator (HTI).

$$
\begin{aligned}
& q_{\text {conv }}+K \frac{\partial T}{\partial x}-q_{r}=\rho C_{P} \frac{\partial T}{\partial t} \quad x=0, \\
& K \frac{\partial T}{\partial x}+q_{r}=\rho C_{P} \frac{\partial T}{\partial t}+K_{s t r} \frac{\partial T}{\partial x} \quad x=L, \\
& K_{s t r} \frac{\partial T}{\partial x}=0 \quad x=L+L_{s t r},
\end{aligned}
$$

where the convection heat flux $\left(q_{\text {conv }}\right)$ can be found as $q_{\text {conv }}=h\left(T_{\infty}-T(0)\right)$. Figure 1 clarifies the Boundary Condition (BC).

Despite the criticisms against applying a onedimensional model to the radiative heat transfer [24,25], it is commonly used for simulating combined conduction and radiation heat transfer [5]. The twoflux methodology can be employed to find the onedimensional radiation heat transfer. This methodology considers two different radiative heat fluxes that move from the hot side to the cold side of the insulator, and vice versa (see Figure 2). The difference between these heat fluxes exhibits radiative heat flux anywhere in the insulator (Eq. (3)):

$$
q_{r}=I_{1}(x)-I_{2}(x) .
$$

Hot to cold and cold to hot radiative heat fluxes can be found by solving the boundary-value differential equation. This first-order nonlinear differential equation system is presented in Eq. (4):

$$
\begin{aligned}
& d I_{1}(x) / d x=-M I_{1}+N I_{2}+P \sigma T^{4}(x), \\
& d I_{2}(x) / d x=-M I_{2}+N I_{1}+P \sigma T^{4}(x),
\end{aligned}
$$

in which:

$$
\begin{aligned}
& P=S_{a}, \\
& N=S_{s}, \\
& M=S_{s}+S_{a} .
\end{aligned}
$$

Scattering and absorbance areas of media are the ratio 


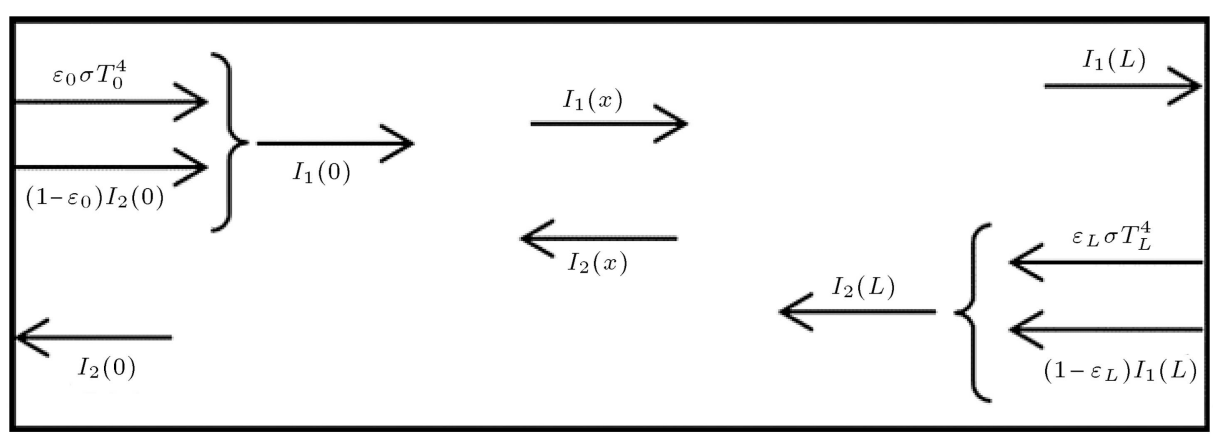

Figure 2. Schematic of internal radiation heat flux.

of the scattered and absorbed light to the total light hitting the media. This paper focuses on materials that have low absorptance area and high scattering area, respectively. Eq. (5) also presents the conditions of the boundary value system. The described BC is deducted from Figure 2 as follows:

$$
\begin{aligned}
& I_{1}(0)=\sigma T^{4}(0) \varepsilon_{0}+\left(1-\varepsilon_{0}\right) I_{2}(0), \\
& I_{2}(L)=\sigma T^{4}(L) \varepsilon_{L}+\left(1-\varepsilon_{L}\right) I_{1}(L) .
\end{aligned}
$$

Radiation heat flux should be calculated at each time step. Then, radiation heat flux should be substituted into the heat transfer equation (Eq. (1)). It is implied that a large amount of computation expense is involved in simulating radiative heat transfer through the thickness of the high-temperature insulator. Considering the non-absorbing media $(P=0, M=N)$ will simplify Eq. (4), which can be analytically solved. In this case, radiative heat transfer is determined through Eq. (6):

$$
q_{r}=\frac{\sigma\left(T^{4}(0)-T^{4}(L)\right)}{1 / \varepsilon_{0}+1 / \varepsilon_{L}-1+S_{s} L} .
$$

Therefore, the heat transfer equation and $\mathrm{BC}$ could be reduced into Eq. (7):

$$
\begin{aligned}
& \rho C_{P} \frac{\partial T}{\partial t}=K \frac{\partial^{2} T}{\partial x^{2}} \\
& q_{\text {conv }}+K \frac{\partial T}{\partial x}-\frac{\sigma\left(T^{4}(0)-T^{4}(L)\right)}{1 / \varepsilon_{0}+1 / \varepsilon_{L}-1+S_{s} L}=\rho C_{P} \frac{\partial T}{\partial t} \\
& x=0, \\
& K \frac{\partial T}{\partial x}+\frac{\sigma\left(T^{4}(0)-T^{4}(L)\right)}{\frac{1}{\varepsilon_{0}}+\frac{1}{\varepsilon_{L}}-1+S_{s} L}=K_{s t r} \frac{\partial T}{\partial x}+\rho C_{P} \frac{\partial T}{\partial t} \\
& x=L .
\end{aligned}
$$

It is possible to present Eq. (7) in another way. The term $q_{r}$ in $\mathrm{BC}$ of this equation is added to the heat differential equation by considering equivalent conductivity. Equivalent radiative thermal conductivity can be written as in Eq. (8):

$$
\begin{aligned}
K_{\text {rad }} & =\frac{\sigma\left(T^{4}(0)-T^{4}(L)\right)}{1 / \varepsilon_{0}+1 / \varepsilon_{L}-1+S_{s} L} \times \frac{L}{T(0)-T(L)} \\
& =\frac{\sigma L(T(0)+T(L))\left(T^{2}(0)+T^{2}(L)\right)}{1 / \varepsilon_{0}+1 / \varepsilon_{L}-1+S_{s} L} \\
& \cong \frac{4 \sigma L T_{\text {mean }}^{3}}{1 / \varepsilon_{0}+1 / \varepsilon_{L}-1+S_{s} L} .
\end{aligned}
$$

Further to the above, Eq. (7) is rewritten as Eq. (9) below:

$$
\begin{aligned}
& \rho C_{P} \frac{\partial T}{\partial t}=\left(K+K_{\text {rad }}\right) \frac{\partial^{2} T}{\partial x^{2}}, \\
& q_{\text {conv }}+\left(K+K_{\text {rad }}\right) \frac{\partial T}{\partial x}=\rho C_{P} \frac{\partial T}{\partial t} \quad x=0, \\
& \left(K+K_{\text {rad }}\right) \frac{\partial T}{\partial x}=K_{\text {str }} \frac{\partial T}{\partial x}+\rho C_{P} \frac{\partial T}{\partial t} \quad x=L .
\end{aligned}
$$

This methodology has been entitled as effective thermal conductivity approximation in different references [26]. It is easy to demonstrate the similarity between Eqs. (7) and (9) in steady state cases when $\partial T / \partial t=$ 0 . Effectiveness of this methodology was validated in several steady state cases. Multi-layer reflective insulators reflect radiation energy using intermediate reflective surfaces. For more details, see Figure 3.

Infinitesimal thickness of the reflective surface would not affect solid thermal conductivity of the insulator. In this case, the heat transfer equation and BCs could be written, as given in Eq. (10). In fact, intermediate surface redirects the major portion of the radiation energy (i.e., $\left.1-\varepsilon_{\text {xint }}\right)$ to the hot side. Another portion of radiation energy (i.e., $\varepsilon_{\text {xint }}$ ) is absorbed by the reflective layer. This point is considered in symbolizing the mathematical model:

$$
\begin{aligned}
& \rho C_{P} \frac{\partial T}{\partial t}=K \frac{\partial^{2} T}{\partial x^{2}}, \\
& q_{c o n v}-\varepsilon \sigma T^{4}(0)+K \frac{\partial T}{\partial x}-\frac{\sigma\left(T^{4}(0)-T^{4}\left(x_{\mathrm{int}}\right)\right)}{1 / \varepsilon_{0}+1 / \varepsilon_{x_{\mathrm{int}}}-1+S_{s} x_{\mathrm{int}}} \\
& \quad=\rho C_{P} \frac{\partial T}{\partial t} \quad x=0
\end{aligned}
$$




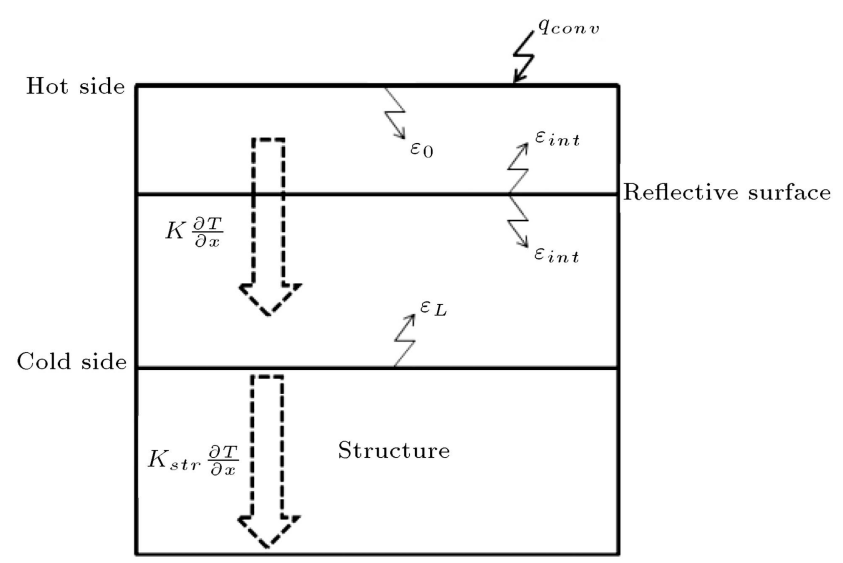

Figure 3. High Temperature Insulator (HTI) with reflective surface.

$$
\begin{aligned}
K \frac{\partial T}{\partial x} & +\frac{\sigma\left(T^{4}(0)-T^{4}\left(x_{\mathrm{int}}\right)\right)}{1 / \varepsilon_{0}+1 / \varepsilon_{x_{\mathrm{int}}}-1+S_{s} x_{\mathrm{int}}} \\
& -\frac{\sigma\left(T^{4}\left(x_{\mathrm{int}}\right)-T^{4}(L)\right)}{1 / \varepsilon_{x_{\mathrm{int}}}+1 / \varepsilon_{L}-1+S_{s}\left(L-x_{\mathrm{int}}\right)}=\rho C_{P} \frac{\partial T}{\partial t} \\
x & =x_{\mathrm{int}}, \\
K \frac{\partial T}{\partial x} & +\frac{\sigma\left(T^{4}\left(x_{\mathrm{int}}\right)-T^{4}(L)\right)}{1 / \varepsilon_{x_{\mathrm{int}}}+1 / \varepsilon_{L}-1+S_{s}\left(L-x_{\mathrm{int}}\right)} \\
& =\rho C_{P} \frac{\partial T}{\partial t}+K_{s t r} \frac{\partial T}{\partial x} \quad x=L .
\end{aligned}
$$

If more than one reflective surface is used, a mathematical model can be developed similarly, which is called primitive model in this paper. As previously stated, the absorption mechanism is not considered in the primitive model.

\subsection{Problem solution}

The numerical explicit methodology was applied to solve the transient 1-D heat transfer equation. The equation and the related BCs were discretized, as given in Eq. (11):

$$
T_{i}^{n+1}=T_{i}^{n}+\frac{\frac{K}{\rho C_{p}} \Delta t}{(\Delta x)^{2}}\left(T_{(i-1)}^{n}-2 T_{i}^{n}+T_{(i+1)}^{n}\right) .
$$

\section{Model assessment}

The mathematical model was evaluated in the transient case. The theoretical model was assessed based on the experimental result of a multi-layer reflective insulator.

Daryabeigi examined an alumina-based insulator with five intermediate reflective surfaces located through the thickness of the multi-layer insulator. The alumina fibrous material was placed between the reflective gold-coated layers. The thermal emissivity of the reflective layers was set to 0.1 , as measured by Darayabeigi [16]. Optical properties of alumina fibrous material were considered as follows [27]:

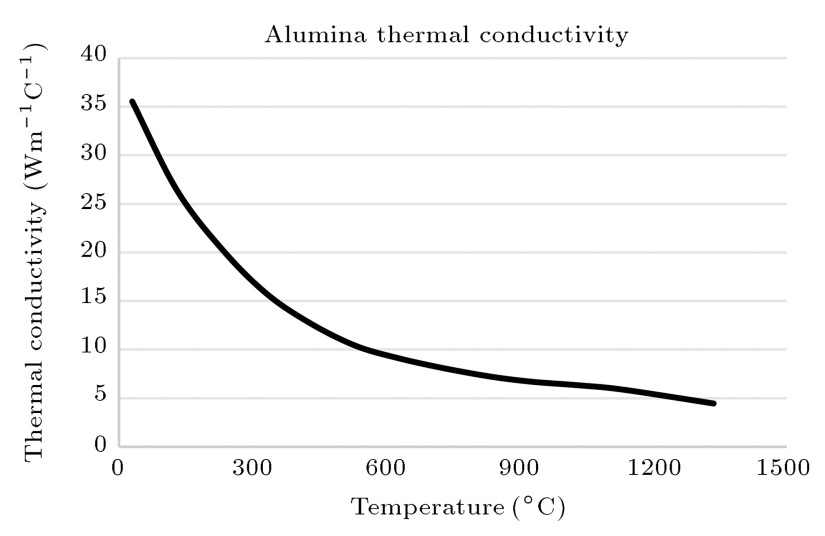

Figure 4. Temperature-dependent thermal conductivity of alumina fibers.

$$
\begin{aligned}
& S_{a}=e \times(1-\omega) \times \rho_{\text {alumina }}, \\
& S_{s}=e \times \omega \times \rho_{\text {alumina }} \times b, \\
& \omega=0.974, \\
& b=0.268, \\
& e=53.017+0.03879 T, \\
& \rho_{\text {alumina }}=24.3 \frac{\mathrm{Kg}}{\mathrm{m}^{3}} .
\end{aligned}
$$

The thermal capacity of alumina was considered 880 $\frac{\mathrm{J}}{\mathrm{kg}^{\circ} \mathrm{C}}$. The solid thermal conductivity of alumina is shown in Figure 4 [28].

The overall thickness of the insulator was 19.14 $\mathrm{mm}$ and reflective surfaces were positioned, as described by Daryabeigi [16]. To consider light scattering along the insulator, scattering area of the alumina was included in the model. However, absorption mechanism was not applied, as expressed previously.

The underlying structure was positioned $13.3 \mathrm{~mm}$ above a plate. The temperature of this plate was kept at $297^{\circ} \mathrm{K}$ using water. An insulator with $13.3 \mathrm{~mm}$ thickness and $24.3 \mathrm{~kg} / \mathrm{m}^{3}$ density was placed between the water-cooled plate and the structure. The temperature of the hot side varied, as shown in Figure 5. The primitive model was used to examine the transient structure temperature. Figure 6 shows the numerical results and compares them with the experimental ones. A high level of discrepancy between the two sets of results was observed, especially after 800 seconds when the hot side of the insulator does not warm, as shown in Figure 5. It appears that ignoring the absorption mechanism leads to erroneous results. The mentioned mechanism is simplified in the following section.

\section{Developed model}

The absorption mechanism gives rise to two different phenomena. It absorbs and simultaneously emits 


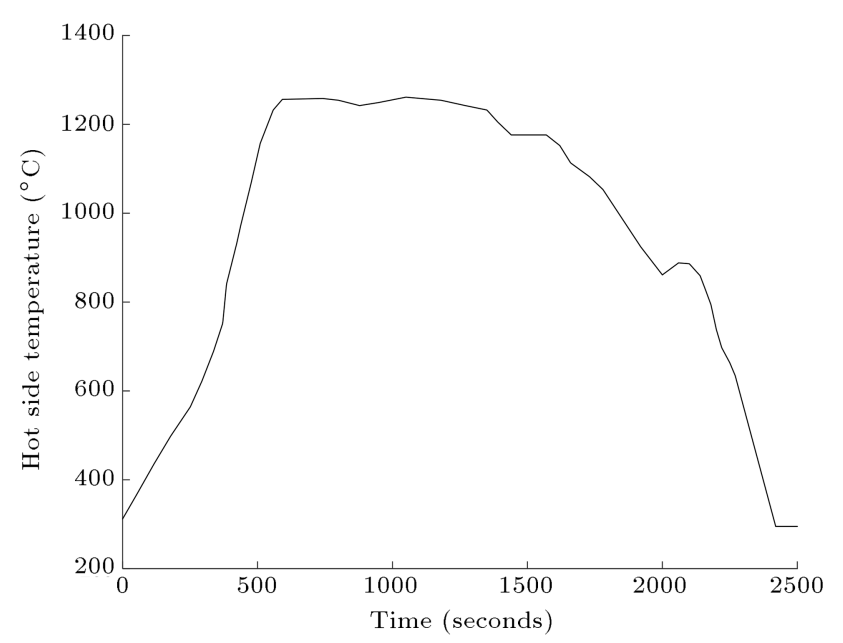

Figure 5. Time-dependent temperature of the external hot side of the insulator.

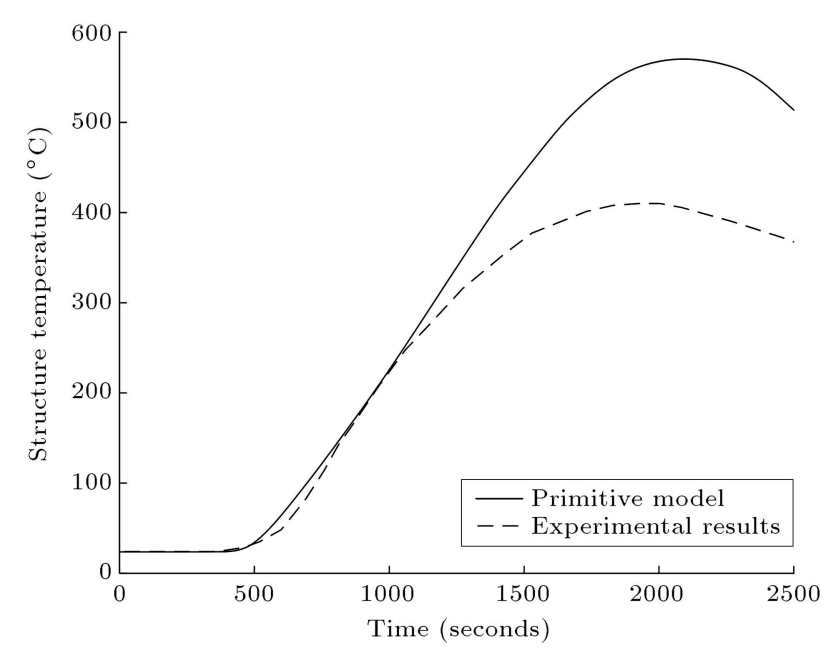

Figure 6. Comparison of the primitive analytical model and experimental model.

radiation heat flux. These two distinct phenomena are presented in Eq. (4).

For simplification, the absorption phenomenon was not considered, while the emittance phenomenon should be taken into account when the hot side did not warm. Therefore, it was supposed that the insulator material located between the reflective layers emitted radiation to the enclosing top and bottom reflective layers. In other words, the energy emitted from each element of the insulator material confined between two reflective layers is divided between its top and bottom layers, as shown in Figure 7. How this division is performed is linked to the variations in the temperature of the external hot side or the internal cold side of the insulator, the distance between the emitting element and top or bottom reflective layer, temperature, and thermal emissivity of the top or bottom reflective layers. This issue is detailed in the following.

A portion of the emitted radiative energy from the

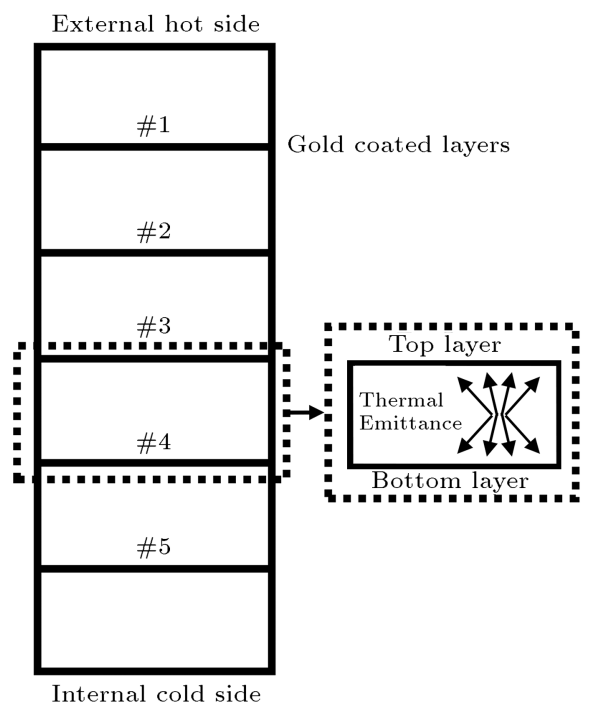

Figure 7. Division of the emitted radiation between the top and bottom surfaces of each layer.

elements located between two near layers absorbed by the top layer, called Top Layer Share (TLS), is the inversely proportional fourth power of the top layer temperature, because the emitted energy is inclined to be absorbed by the cooler layer. TLS is inversely proportional to the distance of the emitting element from the top layer. The insulator medium blocks radiative heat transfer. Therefore, emitted energy will be absorbed by the layer located closer to the emitting element.

Furthermore, TLS is proportional to the thermal emissivity of the Top layer. Emitted energy is mostly absorbed by the layer that has greater thermal emissivity. Bottom Layer Share (BLS) could be achieved in a similar way.

In addition, TLS depends on the rate of temperature variations of the internal cold and external hot sides. This dependency is more complicated than that in the former cases. This dependency corresponds to a condition in which the emitted energy would move toward the side that cools faster. If both of the internal cold and external hot sides get cold, the TLS will be proportional to the rate of variations in the temperature of the external hot side; similarly, the BLS is proportional to the temperature variation rate of internal cold side. In another case, if the external hot side temperature is almost constant and yet, the internal cold side gets warm, TLS will be twice the rate of BLS. In the same conditions, if the internal cold side gets cold, BLS will be twice as large, similarly. However, if the external hot side gets cold and the internal cold side gets warm, TLS will be proportional to the temperature variation rate of the external hot side and also twice the rate of the temperature variations of the internal cold side. In addition, BLS will be proportional to the temperature variation rate of the internal cold side. The analytical 
formulations of TLS and BLS are given as follows:

$$
\begin{aligned}
& P_{T L S}=T_{T O P}^{-4} \times L_{T O P}^{-1} \times \varepsilon_{T O P} \\
& \times \begin{cases}\left|\frac{\partial T_{h o t}}{\partial t}\right| & \frac{\partial T_{h o t}}{\partial t}<0, \frac{\partial T_{\text {cold }}}{\partial t}<0 \\
\left|\frac{\partial T_{h o t}}{\partial t}\right|+2 \times \frac{\partial T_{\text {cold }}}{\partial t} & \frac{\partial T_{h o t}}{\partial t}<0, \frac{\partial T_{\text {cold }}}{\partial t}>0 \\
1 & \frac{\partial T_{h o t}}{\partial t} \cong 0, \frac{\partial T_{\text {cold }}}{\partial t}<0 \\
2 & \frac{\partial T_{h o t}}{\partial t} \cong 0, \frac{\partial T_{\text {cold }}}{\partial t}>0\end{cases} \\
& P_{B L S}=T_{B O T}^{-4} \times L_{B O T}^{-1} \times \varepsilon_{B O T} \\
& \times \begin{cases}\left|\frac{\partial T_{\text {cold }}}{\partial t}\right| & \frac{\partial T_{h o t}}{\partial t}<0, \frac{\partial T_{\text {cold }}}{\partial t}<0 \\
\frac{\partial T_{\text {cold }}}{\partial t} & \frac{\partial T_{h o t}}{\partial t}<0, \frac{\partial T_{\text {cold }}}{\partial t}>0 \\
2 & \frac{\partial T_{h o t}}{\partial t} \cong 0, \frac{\partial T_{\text {cold }}}{\partial t}<0 \\
1 & \frac{\partial T_{h o t}}{\partial t} \cong 0, \frac{\partial T_{\text {cold }}}{\partial t}>0\end{cases} \\
& T L S=P_{T L S} /\left(P_{T L S}+P_{B L S}\right), \\
& B L S=P_{B L S} /\left(P_{T L S}+P_{B L S}\right) .
\end{aligned}
$$

TLS and BLS should be calculated for every element using the thickness of the insulator at each time step. Finally, considering thermal emittance through the thickness of the insulator, the governing equation will be updated to that presented in Eq. (14), in case of one reflective surface.

$$
\begin{aligned}
\rho C_{P} \frac{\partial T}{\partial t} & =K \frac{\partial^{2} T}{\partial x^{2}}-2 \sigma S_{a} T^{4} \\
q_{\text {conv }}-\varepsilon T^{4}(0)+K \frac{\partial T}{\partial x}-\frac{\sigma\left(T^{4}(0)-T^{4}\left(x_{\mathrm{int}}\right)\right)}{1 / \varepsilon_{0}+1 / \varepsilon_{x_{\mathrm{int}}}-1+S_{s} x_{\mathrm{int}}} & \\
& +\int_{x=0}^{x=x_{\mathrm{int}}} 2 \sigma S_{a} T^{4}(x) T L S(x) d x=\rho C_{P} \frac{\partial T}{\partial t} \\
x & =0, \quad \frac{\partial T}{\partial x}+\frac{\sigma\left(T^{4}(0)-T^{4}\left(x_{\mathrm{int}}\right)\right)}{1 / \varepsilon_{0}+1 / \varepsilon_{x_{\mathrm{int}}}-1+S_{s} x_{\mathrm{int}}} \\
& -\frac{\sigma\left(T^{4}\left(x_{\mathrm{int}}\right)-T^{4}(L)\right)}{1 / \varepsilon_{x_{\mathrm{int}}}+1 / \varepsilon_{L}-1+S_{s}\left(L-x_{\mathrm{int}}\right)} \\
& +\int_{x=x_{\mathrm{int}}} 2 \sigma S_{a} T^{4}(x) B L S(x) d x
\end{aligned}
$$

$$
\begin{gathered}
\quad+\int_{x=x_{\mathrm{int}}}^{x=L} 2 \sigma S_{a} T^{4}(x) T L S(x) d x=\rho C_{P} \frac{\partial T}{\partial t} \\
x=x_{\mathrm{int}}, \\
K \frac{\partial T}{\partial x}+\frac{\sigma\left(T^{4}\left(x_{\mathrm{int}}\right)-T^{4}(L)\right)}{1 / \varepsilon_{x_{\mathrm{int}}}+1 / \varepsilon_{L}-1+S_{s}\left(L-x_{\mathrm{int}}\right)} \\
+\int_{x=x_{\mathrm{int}}} 2 \sigma S_{a} T^{4}(x) B L S(x) d x=\rho C_{P} \frac{\partial T}{\partial t} \\
+K_{s t r} \frac{\partial T}{\partial x} \quad x=L .
\end{gathered}
$$

The explicit numerical methodology was used to solve Eq. (13). As shown in Figure 8, the application of this model reduces the errors of the primitive model. The error of the developed analytical model is less than $4 \%$. This model can properly predict the maximum temperature of the structure and its occurrence time. Therefore, the model can be applied to the earlier stages of design.

The main assumption of this model is ignoring the effect of the absorption mechanism on radiation heat transfer to the structure.

Streed et al. introduced and experimentally verified a model for equivalent radiative thermal conductivity of the multi-layer insulation systems. This model considers the absorption mechanism effect on radiative heat transfer, as presented in Eq. (14) [6]:

$$
K_{r}=\frac{\sigma\left(T(0)^{2}+T(L)^{2}\right)(T(0)+T(L)) L}{\left(2 S_{s}+S_{a}\right) \frac{L}{2}+(N-1)\left(\frac{2}{\varepsilon}-1\right)} .
$$

Eq. (14) shows that the absorption mechanism impacts the equivalent radiative thermal conductivity similar

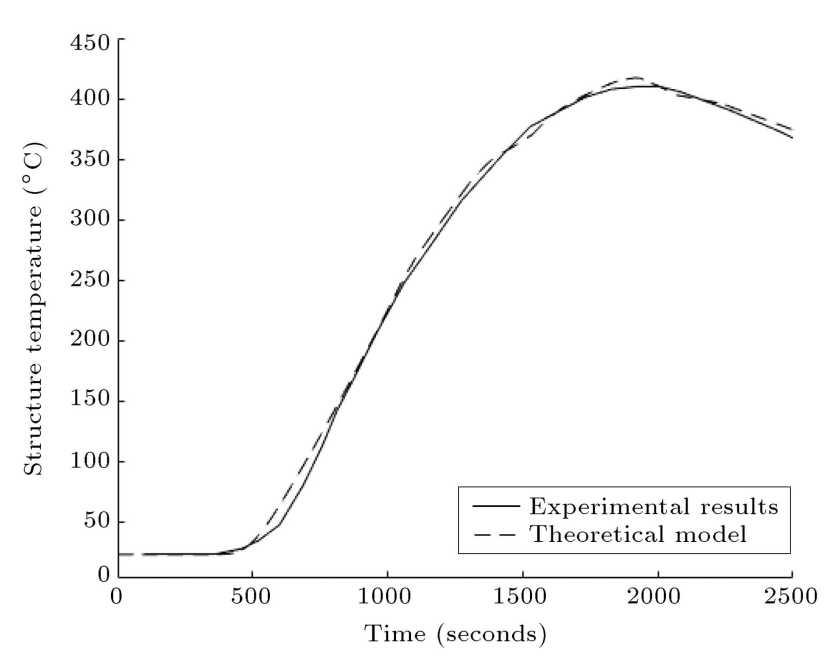

Figure 8. Time-dependent structure temperature when experimental thermal load is applied. 


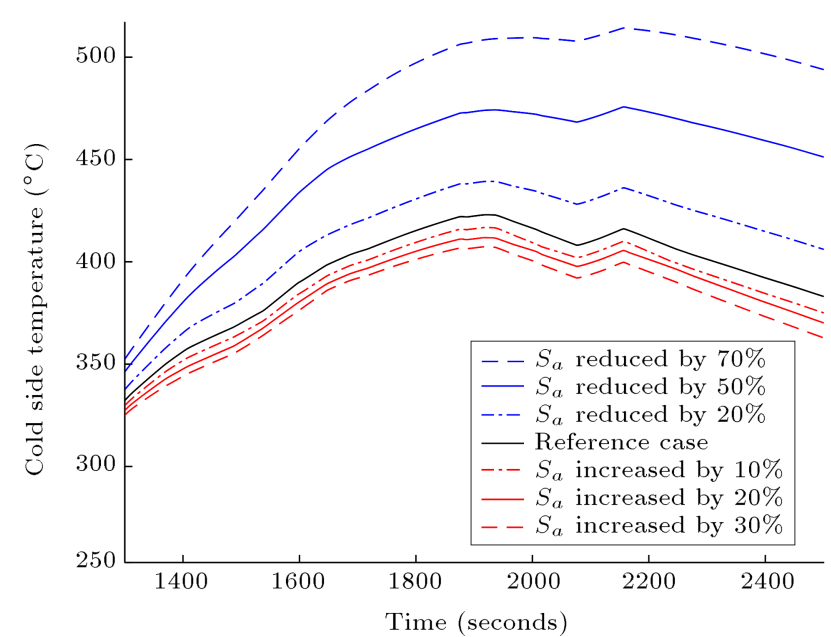

Figure 9. Effect of absorption area variations on the structure temperature.

to the scattering one. According to Eqs. (11) and (14), it can be deduced that the absorption mechanism will reduce the equivalent thermal conductivity only by less than $0.5 \%$. Therefore, it is perfectly acceptable to not consider the effect of the absorption mechanism. The following figure shows the effect of absorption area variations on the structure temperature. As expected, structure temperature increases if the absorption area was reduced. On the contrary, it is expected that increasing the absorption area would decrease temperature of the structure.

According to Figure 9, it is clear that increasing the absorption area by $30 \%$ leads to a decrease in the maximum structure temperature by $3.5 \%$. However, if the absorption area is reduced to $30 \%$ of its initial value, the structure temperature will be elevated by about $22 \%$. Moreover, according to Figure 6 , it can be deduced that if the absorption area is not considered, the structure temperature would be increased by as high as $45 \%$. Another important issue depicted in Figure 9 is that the absorption area shortens the time required to reach the maximum temperature by 200 seconds.

\section{Conclusion}

A quasi-mathematical model was developed to simulate combined conduction and radiation heat transfer through the thickness of a high-temperature multi-layer reflective insulator. The model was developed based on the effective thermal conductivity approximation. It can overcome shortcomings of the effective thermal conductivity approximation in transient thermal load cases. This model considered the thermal emittance of insulator elements through the thickness of the insulator. An engineering analysis was carried out to estimate how the emitted energy would be split between the top and bottom reflective layers. Then, the numerical result of the model was compared with the experimental results in the transient case. The theoretical result showed good agreement with experimental results. Maximum temperature and time of occurrences were predicted by an error less than $4 \%$. In comparison to the two-flux and diffusion models, the developed model was simpler and required less computational cost, which is an important issue in the earlier stages of the design process. Furthermore, this methodology outperformed other methods in terms of precision in simulating transient heat transfer.

\section{Nomenclature}

$\rho$

$C_{P} \quad$ Specific heat capacity of the insulator

$K \quad$ Thermal conductivity of the insulator

$T \quad$ Temperature of the insulator

$b \quad$ Back scattering fraction

$t \quad$ Time

e Specific extinction

$x \quad$ Location

$q_{r} \quad$ Radiative heat flux

$L \quad$ Insulator thickness

$L_{\text {str }} \quad$ Structure thickness

$q_{\text {conv }} \quad$ Convection heat flux

$T_{\infty} \quad$ Ambient temperature

$h \quad$ Convection coefficient

$I_{1}(x) \quad$ Radiative heat flux from the hot side to the cold side

$I_{2}(x) \quad$ Radiative heat flux from the cold side to the hot side

$K_{s t r} \quad$ Structure Thermal conductivity

$K_{r} \quad$ Equivalent radiative thermal conductivity

$N \quad$ Number of layers

$S_{s} \quad$ Scattering area

$S_{a} \quad$ Absorbance area

$\sigma \quad$ Stefan-Boltzmann constant

$\varepsilon_{L} \quad$ Radiative emissivity of the hot side

$\varepsilon_{0} \quad$ Radiative emissivity of the cold side

$\omega \quad$ Albedo of scattering

$x_{\text {int }} \quad$ Intermediate reflective surface location

$\varepsilon_{\text {int }} \quad$ Intermediate reflective surface emissivity

TLS Top Layer Share

BLS Bottom Layer Share

$T_{T O P} \quad$ Top layer temperature

$T_{B O T} \quad$ Bottom layer temperature

$L_{T O P} \quad$ Distance between emitting element and top layer 
$L_{B O T} \quad$ Distance between emitting element and bottom layer

$\varepsilon_{T O P} \quad$ Top layer thermal emissivity

$\varepsilon_{B O T} \quad$ Bottom layer thermal emissivity

$T_{\text {cold }} \quad$ Cold side temperature

$T_{\text {hot }} \quad$ Hot side temperature

$T_{\text {mean }} \quad$ Mean temperature of insulator

\section{References}

1. He, Y.L. and Xie, T. "Advances of thermal conductivity models of nanoscale silica aerogel insulation material", Applied Thermal Engineering, 81, pp. 28$50(2015)$.

2. Petrov, V.A. "Combined radiation and conduction heat transfer in high temperature fiber thermal insulation", International Journal of Heat and Mass Transfer, 40(9), pp. 2241-2247 (1997).

3. Cowart, K. and John, O. "TCAT-A tool for automated thermal protection system design", Space 2000 Conference and Exposition (2000).

4. Bai, D. and Fan, X.J. "Integrated design system for dynamic thermal protection system sizing", Proceedings of the Institution of Mechanical Engineers, Part G: Journal of Aerospace Engineering, 221(5), pp. 883892 (2007).

5. Cleland, J. and Francesco, L. "Thermal protection system of the space shuttle", Research Triangle INST Research Triangle NC (1989).

6. Streed, E.R., Cunningtont, G.R., and Zierman, C.A. "Performance of multilayer insulation systems for the $300^{\circ}$ to $800^{\circ} \mathrm{K}$ temperature range", Thermophysics and Temperature Control of Spacecraft and Entry Vehicles, Academic Press, pp. 735-771 (1966).

7. Pietrak, K. and Wiśniewski, T.S. "A review of models for effective thermal conductivity of composite materials", Journal of Power Technologies, 95(1), pp. 14-24 (2014).

8. Lacroix, C., Ramany Bala, P., and Feidt, M. "Evaluation of the effective thermal conductivity in metallic porous media submitted to incident radiative flux in transient conditions", Energy Conversion and Management, 40(15-16), pp. 1775-1781 (1999).

9. Hao, J.H., Chen, Q., and Hu, K. "Porosity distribution optimization of insulation materials by the variational method", International Journal of Heat and Mass Transfer, 92, pp. 1-7 (2016).

10. Yu, H.T., Liu, D., Duan, Y.Y., and Wang, X.D. "Theoretical model of radiative transfer in opacified aerogel based on realistic microstructures", International Journal of Heat and Mass Transfer, 70, pp. 478485 (2014).

11. Mendes, M.A., Talukdar, P., Ray, S., et al. "Detailed and simplified models for evaluation of effective thermal conductivity of open-cell porous foams at high temperatures in presence of thermal radiation", International Journal of Heat and Mass Transfer, 68, pp. 612-624 (2014).

12. Shuyuan, Z., Boming, Z., and Shanyi, D. "Effects of contact resistance on heat transfer behaviors of fibrous insulation", Chinese Journal of Aeronautics, 22(5), pp. 569-574 (2009).

13. Arambakam, R., Vahedi Tafreshi, H., and Pourdeyhimi, B. "Modeling performance of multi-component fibrous insulations against conductive and radiative heat transfer", International Journal of Heat and Mass Transfer, 71, pp. 341-348 (2014).

14. Wei, K., Cheng, X., Mo, F., et al. "Design and analysis of integrated thermal protection system based on lightweight $\mathrm{C} / \mathrm{SiC}$ pyramidal lattice core sandwich panel", Materials \& Design, 111, pp. 435-444 (2016).

15. Torabi, A., Abedian, A., Farsi, M.A., et al. "Axiomatic design of a reflective multilayer high-temperature insulator", Proceedings of the Institution of Mechanical Engineers, Part G: Journal of Aerospace Engineering, 233(2), pp. 457-471 (2019).

16. Daryabeigi, K. "Thermal analysis and design optimization of multilayer insulation for reentry aerodynamic heating", Journal of Spacecraft and Rockets, 39(4), pp. 509-514 (2002).

17. Ji, T., Zhang, R., Sunden, B., et al. "Investigation on thermal performance of high temperature multilayer insulations for hypersonic vehicles under aerodynamic heating condition", Applied Thermal Engineering, 70(1), pp. 957-965 (2014).

18. Daryabeigi, K. "Analysis and testing of high temperature fibrous insulation for reusable launch vehicles", 37th Aerospace Sciences Meeting and Exhibit, USA, AIAA, 99-1044 (1999).

19. Daryabeigi, K. "Heat transfer in high-temperature fibrous insulation", Journal of Thermophysics and Heat Transfer, 17(1), pp. 10-20 (2003).

20. Larkin, B.K. and Churchill, S.W. "Heat transfer by radiation through porous insulations", AIChE Journal, 5(4), pp. 467-474 (1959).

21. Howell, J.R., Menguc, M.P., and Siegel, R., Thermal Radiation Heat Transfer, CRC press (2015).

22. Fang, W.Z., Zhang, H., Chen, L., et al. "Numerical predictions of thermal conductivities for the silica aerogel and its composites", Applied Thermal Engineering, 115, pp. 1277-1286 (2017).

23. Curry, D.M., Space Shuttle Orbiter Thermal Protection System Design and Flight Experience, NASA TM104773 (1993)

24. Lee, S.C. "Effect of fiber orientation on thermal radiation in fibrous media", International Journal of Heat and Mass Transfer, 32(2), pp. 311-319 (1989).

25. Lee, S.C. "Scattering phase function for fibrous media", International Journal of Heat and Mass Transfer, 33(10), pp. 2183-2190 (1990). 
26. Aegerter, M.A., Leventis, N., and Matthias M.K., Eds., Aerogels Handbook, Springer Science \& Business Media (2011).

27. Daryabeigi, K., Miller, S.D., and Cunnington, G.R. "Heat transfer in high-temperature multilayer insulations", Proceedings of the 5th European Workshop on Thermal Protection Systems and Hot Structures, ESTEC, Noordwijk, the Netherlands, May 17-19 (2006).

28. Auerkari, P., Mechanical and Physical Properties of Engineering Alumina Ceramics, Espoo: Technical Research Centre of Finlan (1996).

\section{Biographies}

Amin Torabi was born in Tehran, Iran in 1986. He received BS degree in Aerospace Engineering and MSc degree in Structural Orientation of Aerospace Engineering from the Sharif University of Technology, Tehran, Iran in 2008 and 2011 respectivily, and PhD degree in Structural Orientation of Aerospace Engineering from the Sharif University of Technology and Aerospace Research Institute, Tehran, Iran in 2018. His current research interests include axiomatic design, systematic design, structural design, thermal protection systems, thermal barrier coatings, functionally graded materials, structural and thermal analysis, and radiation heat transfer. He has contributed more than 2 journal papers and 3 conference papers.

Ali Abedian is an Associate Professor of Aerospace Engineering at Sharif University of Technology (SUT), Tehran, Iran. His fields of research interest include thermo/mechanical analysis of composite materials. He also works on the rejuvenation of aged metallic and composite aero-structures using composite materials. His field of expertise also involves application of composite materials to oil/gas/petrochemical industries.

Mohammad Ali Farsi received the BE, ME, and $\mathrm{PhD}$ degrees from Amirkabir University of Technology, Tehran, Iran in 1999, 2002, and 2008, respectively. He joined Aero Space Institute (Ministry of Science, Research and Technology) in 2006 and he is currently an Associate Professor. His main areas of research interest are reliability, safety, maintainability, and system engineering. 